\title{
Reduced Length of Hospital Stay in Laparoscopic Hysterecto- my after Implementation of Enhanced Recovery after Surgery (ERAS) Protocol: a Pilot Program Result
}

\author{
Jeffrey Huang ${ }^{1 *}$, Jessica Vaught ${ }^{2}$ and Lea Meir ${ }^{3}$ \\ ${ }^{1}$ Department of Anesthesiology, Anesthesiologists of Greater Orlando, Florida \\ ${ }^{2}$ Obstetrics/Gynecology, Winnie Palmer Hospital, Orlando, Florida \\ ${ }^{3}$ University of Central Florida, Orlando, Florida
}

\begin{abstract}
Background: Enhanced Recovery After Surgery (ERAS) is a cohesive perioperative surgical care program, reliant of evidence based medicine, which is utilized to enhance patient care. This is done by minimizing surgical stress, improving functional recovery, reducing complications, as well as facilitating a more rapid hospital discharge. This study analyzed the influence of implementing an enhanced recovery following surgery protocol on patient outcomes in women who have received a laparoscopic hysterectomy procedure. We hypothesized that adoption of an Enhanced Recovery After Surgery (ERAS) protocol would reduce hospital length of stay when contrasted to traditional care.
\end{abstract}

Methods: This study was designed as a retrospective cohort study. Patients assigned to the Enhanced Recovery protocol were analyzed against historic controls from Jan 1, 2014 to March 30, 2014, prior to the implementation of the enhanced recovery protocol. No substantial variations in technology, surgical techniques, took place between the study period and the period from which historic controls were drawn. Outcome measurements included the percentage of patients who were admitted to the hospital after surgery (length of stay), Readmission rates, and complications.

Results: The percentage of patients admitted to hospital after surgery (length of stay) was significantly reduced after ERAS implementation $(70 \%$ vs. $33 \%)(P<0.05)$. This contrasts to the readmission rates and complications which were not significantly altered after ERAS implementation.

Conclusion: This small sample sized cohort study demonstrated that the ERAS program in laparoscopic hysterectomy significantly reduces the percentage of patients admitted to hospital after surgery (length of stay), with no increase in patient readmission rates or complications.

\section{Keywords}

Enhanced recovery after surgery, Hysterectomy, Length of hospital stay, Complications, Readmissions

\section{Introduction}

Hysterectomy is the most frequently performed surgical procedure for women in the United States. Approximately 600,000 hysterectomies are performed annually. Abdominal hysterectomies are performed in $66 \%$ of cases, vaginal hysterectomies in $22 \%$ of cases, and laparoscopic hysterectomies in $12 \%$ of cases [1].

Laparoscopic surgery for hysterectomy offers many advantages including a faster return to normal activity, shorter duration of hospital stay, lower intraoperative blood loss, and fewer wound infections [2]. Laparoscopic hysterectomy may be performed safely as an out patient procedure, offering a convenience to patients. This is reflective in the fact that the current number of inpatient hysterectomies performed in the United States has declined [3].

Enhanced Recovery After Surgery (ERAS) is a cohesive perioperative surgical care program, reliant of evidence based medicine, which is utilized to enhance patient care. Implementation of ERAS in gynecologic surgery was associated with positive improvement of the clinical outcomes [4]. Few studies had been conducted to examine the results of same day discharge after surgery in laparoscopic hysterectomy.

The goal of the study was to assess clinical effectiveness of implementing ERAS protocol patient after laparoscopic hysterectomy. We hypothesized that adoption of an ERAS protocol reduces hospital LOS, complications, when compared with traditional care.

\section{Methods}

An Enhanced Recovery After Surgery protocol was developed by an inter disciplinary team. This protocol was based on the most current recommendations from the literature and accounted for the unique issues presented by gynecologic surgical patients (Appendix 1). Enhanced Recovery Protocol Pilot Program was instituted on April 1, 2014, and was used for all patients who under went laparoscopic hysterectomy by the single surgeon (JV) from that date forward.

This was a retrospective cohort study. After obtain- 
Table 1: Patient demographic data.

\begin{tabular}{|l|l|l|l|}
\hline & Before ERAS (21) mean & After ERAS (30) mean & P value \\
\hline Age (years) & $46(27-68)$ & $42(23-63)$ & 0.196 \\
\hline Ht (cm) & $160.5(149-175)$ & $162.4(147-161)$ & 0.213 \\
\hline Wt (kg) & $92.6(57-139)$ & $79.3(57-135)$ & 0.086 \\
\hline Surgical time (minutes) & $118(63-200)$ & $110(40-205)$ & 0.893 \\
\hline EBL (ml) & $77(25-400)$ & $74(20-300)$ & 0.709 \\
\hline PACU time (I) (minutes) & $128(62-218)$ & $120(60-207)$ & 0.659 \\
\hline
\end{tabular}

$\mathrm{Ht}$ : Height, Wt: Weight, EBL: Estimated Blood Loss, ${ }^{*} \mathrm{P}<0.05$

Table 2: Complications, mortality, readmission.

\begin{tabular}{|l|l|l|}
\hline & Before ERAS & After ERAS \\
\hline Complications & 1 & 0 \\
\hline Mortality & 0 & 0 \\
\hline Readmission & 0 & 0 \\
\hline
\end{tabular}

ing Institutional Review Board approval, enhanced recovery patients were then compared with consecutive historic controls from Jan 1, 2014 to March 30, 2014. These historic controls were taken during a time period prior to when the enhanced recovery was implemented. No significant changes in technology, surgical techniques, took place between the study period and the period from which historic controls were drawn.

Patients in the ERAS group were educated in the preoperative surgical clinic about the ERAS pathway and were encouraged to ask questions. A patient education video was produced to help patients make an educated and careful decision about their surgery and become familiar with their surgery journey. The video was posted in YouTube and patients were required to watch the video before surgery. Patients were allowed to drink clear fluids until 2 hours pre operatively. The ERAS Protocol was additionally explained to supportive staff. Education for this was done during anesthesia staff meetings which involved both Anesthesia and OR staff.

All patients received general anesthesia (midazolam, fentanyl, famotidine, \pm scopolamine patch, propofol, rocunonium, sevoflurane or desflurane) and oral endotracheal tube. Intraoperative IV opioids were given after induction of anesthesia at the clinician's discretion. Orogastric tubes, placed after the induction of anesthesia, were removed at the end of the procedure.

Outcomes measurements included: the percentage of patients who were admitted to hospital after surgery (length of stay), readmission rates, and complications. Complications were documented in the patient records as wound complications, bowel injuries, urological injuries, or hemorrhage.

Following the laparoscopic hysterectomy procedure, the patients were permitted to be discharged home on the same day or could remain in the hospital overnight. Reducing the number of patients' which stayed overnight, allows for the reduction of the patient's length of stay, improves the hospital bed utilization, and decreas-

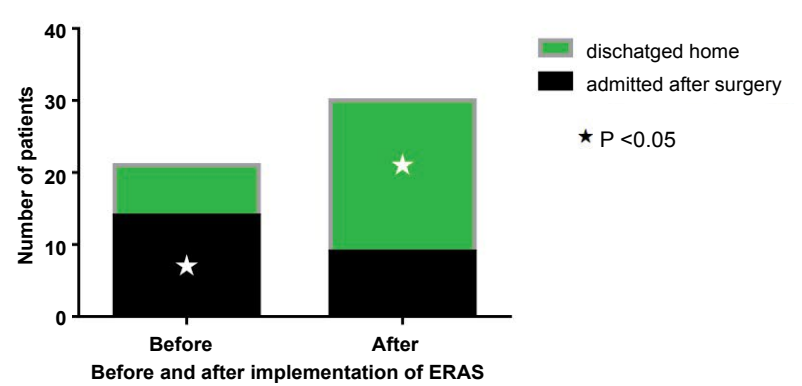

Figure 1: Number of patients admitted to hospital after surgery.

es consumption of hospital resources, thereby reducing costs. Therefore, the number of patients who were discharged home after surgery was used as the measurement of length of stay.

Data was analyzed with Wilcoxon rank-sum test for two independent groups on continuous variables. Statistical significance was defined by a $p$-value less than 0.05 .

\section{Results}

Data was compared between 30 patients who underwent laparoscopic hysterectomy and who were enrolled in the ERAS program Vs. 21 control patients treated in the pre-ERAS months. Patient demographic data included age, height, weight, surgical time, EBLPACU time were statistically comparable between the two groups (Table 1).

The percentage of patients who were admitted to the hospital after surgery (length of stay) were significantly reduced after ERAS implementation (70\% Vs. $33 \%)(P<0.001)$ (Figure 1). Major reasons for a patient's admission following surgery include: comorbid medical conditions, pain, or social factors. Of these constituting reasons, the majority of patients in this study were admitted due to pain, late care, or patient request.

Following the implementation of The Eras protocol in the control gorup, the IV narcotic requirement was lowered after patients were admitted the the floor. 5 patients requested IV narcotics for pain control in the floor, while no patient in ERAS group needed IV narcotics in the floor. However, the incidence of post operative nausea and vomiting in PACU was not found to be statistically different between the two groups. The control group had 8 patients who had post-operative 
nausea/vomiting and needed antiemetic medications in PACU, while the ERAS group had 12 patients.

Readmission mortality, and complications were not significantly altered as a result of ERAS implementation (Table 2).

\section{Discussion}

Enhanced Recovery After Surgery (ERAS) has proven to be successful in bettering the quality and efficiency of surgical management. It has been strongly correlated with a decrease in the hospital stay duration, readmission rate, and the number of repeat operations. In addition to this, it is linked with a decrease in the morbidity and mortality and has improved pain control, cost containment, and patient satisfaction [5-12].

ERAS enhances postoperative recovery by reducing the stress response associated with surgery [13]. Other ERAS studies have been shown to illustrate this point. For example, a ERAS study conducted on patients undergoing colorectal surgery demonstrated the marked reduction in stress response following surgeries which followed ERAS protocols. Cortisol levels were found to be significantly reduced in the ERAS group compared to matched controls.

Generally, excess stress involves a significant release of hormones which may consequently disturb metabolism and cause alterations in insulin resistance, gluconeogenesis, protein catabolism, and lipolysis. ERAS protocols which involve minimally invasive surgeries typically spare postoperative opioids and have been known to enhance metabolic recovery.

Key elements common to all enhanced recovery pathways include: preoperative patient education, reduction of preoperative fasting, omission of bowel preparation, prophylaxis against thromboembolism perioperative normovolemia, standard anesthesia protocol, postoperative nausea and vomiting prevention, laparoscopic surgery, limited use of nasogastric tubes and drains, early removal of urinary catheters, multimodal analgesia to minimize opiate consumption, early postoperative mobilization, postoperative ileus prevention, and early oral in take.

The benefits of ERAS have been demonstrated in patients undergoing gynecological surgeries [14-20]. Implementation of ERAS was not found to be associated with read mission rates or complications. Instead this data along with the results from this retrospective cohort study support the implementation of ERAS pathway to patients undergoing laparoscopic hysterectomy in order to both reduce length of hospital stay as well as improve patient satisfaction.

Patient education given to patients before the procedure about surgical and anesthetic procedures may reduce fear and anxiety and improve postoperative recovery and accelerate hospital discharge [21,22]. All patients were assessed by Pre Admission Testing (PAT) team member before surgery. PAT improves efficiency by enabling early review of the medical records of complex cases, and ensures appropriate examinations and tests that are conducted. PAT can refer patients to other specialists for further evaluation if necessary. Our surgery patient education video was made to allow patients and their relatives the opportunity to familiarize themselves with the environment and to know staff who would provide for their peri-operative care.

Over the past decade, the number of days surgeries that were performed in Canada has increased by $30 \%$, and the number of procedures which require hospital admission has dropped by almost $17 \%$ [23]. The day surgery patients in The US included patients who were discharged on the day of surgery and those who stayed overnight. Same day discharge provides the advantage of avoiding an overnight stay, allowing for decreased utilization for hospital ward and bed. In our facility, 23-hr stay patients remain in the regular surgical ward and require the same care as the other in patients. Therefore, reducing the number of patients who stay overnight can reduce the length of hospital stay, the consumption of hospital resources, as well as hospital costs. Two studies analyzed the cost savings of reduced length of stay after implementation of ERAS protocol for women receiving open or laparoscopic hysterectomies. The first study noted a cost saving of \$495 per patient, the second noted a 27 bed day were saved amongst 31 patients implemented in ERAS protocol over a 2 month time period, equating to a saving of \$285 per patient [24]. While these studies demonstrate considerable cost effectiveness of ERAS implementation, recent studies have additionally shown that same day discharge of patients under going hysterectomy has proven to be safe, carrying with it a low morbidity and low readmission rates as well [24].

Hospital readmission contributes greatly to health care costs leading to the large summation of approximately 17 billion dollars yearly. Same day discharge may even be thought to reduce readmission rates when compared with patients admitted overnight. One significant risk factor for readmission rates within 90 days post-surgery includes the length of stay in the hospital greater than one day compared to one which is less than 24 hours [40\% versus 23\%, $P=0.04$ ] [25]. This study demonstrated that the ERAS program implemented on laparoscopic hysterectomy significantly reduces the number of patients who stayed overnight in hospital after surgery.

Use of multimodal analgesia is strongly recommended for postoperative pain management. Nonsteroidal 
Anti-Inflammatory Drugs (NSAIDs) have been widely studied for gynecologic surgery. It has been shown that NSAIDs can effectively reduce pain and opioid consumption and improve patient satisfaction $[26,27]$. A combination of NSAID and acetaminophen is more effective than either drug alone [28]. A recent systematic review found that administration of gabapentin in prior to surgery for abdominal hysterectomy significantly reduced post-operative pain, opioid consumption and side effects [29]. This study demonstrated that use of multimodal analgesia can reduce post operative IV narcotics requirement.

Postoperative Nausea Vomiting (PONV) is a common complication of surgery. The incidence of PONV can be as high as $70-80 \%$ in high risk patients [30]. The risk factors include age $<50$ years, gynecologic surgery, laparoscopic surgery, female gender, history of PONV or motion sickness, non-smoking, use of volatile anesthetics, long duration of anesthesia, postoperative opioid use, and obesity [30,31]. A multimodal approach to PONV prevention has become the standard of care. Our institute has applied this multimodal approach years before ERAS implementation. Therefore, the study revealed that the incidence of postoperative nausea vomiting was not different before and after ERAS program implementation.

This pilot study demonstrated that there was a reduced length of stay following implementation of ERAS protocol in patients undergoing laparoscopic hysterectomies. The design of the study involved gathering consecutive historical controls from January 2014 to March 2014 and comparing them with patients undergoing ERAS Protocol. While it is likely that the extrapolations of this study are widely generalizable as they have been for the implementation of other ERAS protocol implementations, the patients were not randomly assigned and were instead studied as a retrospective cohorts. Further study of large scale implementation in a randomized control trial may instead offer greater conclusive evidence as to the external validity of the study.

There are three important limitations of the current study that must be considered in interpreting the results presented. First, the number of subjects in the two groups was relatively small. This pilot program was intended to provide a platform for the organization to test the protocol, prove value before spending a significant amount of time and resource on large-scale implementation. Second, the cohort study methodology was selected to evaluate the feasibility, safety of the ERAS pathway among a homogenous group of patients over a relatively short period of time. This study was designed to be a pilot study, these weaknesses discussed above are not applicable as they would be in a randomized control trial.
Lastly, clarifying the exact reason for admissions remained a difficult task as this study is retrospective in nature. For this reason patients cannot be clearly identified if they were admitted to pain, comorbid medical conditions, or social factors which are the most common reason to be readmitted.

\section{Conclusion}

Implementation of the ERAS Protocol in laparoscopic hysterectomy has greatly reduced the length of stay of patients admitted to the hospital following surgery, with no change in the either the amount of readmissions or complications.

\section{Acknowledgement}

The abstract was presented in 2015 American Congress of Obstetricians and Gynecologists annual meeting in San Francisco, US.

Corresponding Author: Jeffrey Huang, Department of Anesthesiology, Anesthesiologists of Greater Orlando, 2699 Lee Road, Suite 510, Winter Park, FL 32789, Florida, E-mail: jeffreyhuangmd@gmail.com

Editor: Yuan-Xiang Tao, Ph.D., M.D., Professor and Vice Chair, Director, Center for Pain Medicine Research Department of Anesthesiology, Editor in Chief, The Translational Perioperative and Pain Medicine, Rutgers, The State University of New Jersey, New Jersey Medical School, 185 S. Orange Ave., MSB, E-661 Newark, NJ 07103. Phone: 973-972-9812 (O) Email: yt211@njms.rutgers.edu

\section{Additional publication details}

Journal short name: Transl Perioper \& Pain Med

Received Date: August 29, 2017

Accepted Date: September 19, 2017

Published Date: October 02, 2017

Citation: Huang J, Vaught J, Meir L. Reduced Length of Hospital Stay in Laparoscopic Hysterectomy after Implementation of Enhanced Recovery after Surgery (ERAS) Protocol: a Pilot Program Result. Transl Perioper \& Pain Med 2017; 4 (4):18-23.

Copyright: (C) 2017 Huang J, et al. This is an open-access article distributed under the terms of the Creative Commons Attribution License, which permits unrestricted use, distribution, and reproduction in any medium, provided the original author and source are credited. 


\section{References}

1. Wu JM, Wechter ME, Geller EJ, Nguyen TV, Visco AG. Hysterectomy rates in the United States, 2003. Obstet Gynecol 2007;110:1091-5.

2. Nieboer TE, Johnson N, Lethaby A, Tavender E, Curr E, Garry R, et al. Surgical approach to hysterectomy for benign gynaecological disease. Cochrane Database of Systematic Reviews 2009.

3. Wright J,Herzog T, Tsui J,Ananth C, et al. Nationwide Trends in the Performance oflnpatient Hysterectomy in the United States.Obstet Gynecol 2013; 122: 233-241.

4. Kalogera E, Bakkum-Gamez JN, Jankowski CJ, Trabuco E, Lovely JK, Dhanorker S, Grubbs PL, Weaver AL, Haas LR, Borah BJ, Bursiek AA, Walsh MT, Cliby WA, Dowdy SC. Enhanced recovery in gynecologic surgery. Obstet Gynecol 2013;122:319-2

5. Khoo CK, Vickery CJ, Forsyth N,Vinall NS, Eyre Brook IA. A prospective randomized controlled trial of multimodal perioperative management protocol in patients undergoing elective colorectal resection for cancer. Ann Surg 2007;245:867-72.

6. Kehlet H, Dahl JB. Anaesthesia, surgery, and challenges in postoperative recovery. Lancet 2003;362:1921-8.

7. Wind J, Polle SW, Fung Kon Jin PH, etal. Systematic review of enhanced recovery programmes in colonic surgery. Br J Surg 2006;93:800-9.

8. Gouvas N, Tan E, Windsor A, Xynos E, Tekkis PP. Fast track vs standard care in colorectal surgery: a meta analysis update. Int J Colorectal Dis 2009;24:1119-31.

9. Huang J. Economic Benefits of Enhanced Recovery After SurgeryJ Med Pract Manage. 2016;31:388-91.

10. Huang J: Enhanced recovery after surgery (ERAS) protocols and perioperative lung protection. J Anesth Perioper Med 2014; 1: 50-56

11. Zhu S, Qian W, Jiang C, Ye C, Chen X. Enhanced recovery after surgery for hip and knee arthroplasty: a systematic review and meta-analysis. Postgrad Med J 2017.

12. Pang KH, Groves R, Venugopal S, Noon AP, Catto JWF. Prospective Implementation of Enhanced Recovery After Surgery Protocols to Radical Cystectomy. Eur Urol 2017.

13. Ren, L., Zhu D, Wei Y, Pan X, Liang L, Xu J, Zhong Y, Xue Z, Jin L, Zhan S, Niu W, Qin X, Wu Z, Wu Z. "Enhanced Recovery After Surgery (ERAS) program attenuates stress and accelerates recovery in patients after radical resection for colorectal cancer: a prospective randomized controlled trial." World J Surg 2012;36: 407-414.

14. Ottesen M, Sorensen M, Kehlet H, Ottesen B. Short convalescence after vaginal prolapse surgery.Acta Obstet Gynecol Scand 2003;82:359-66.

15. Ottesen $M$, Sorensen M,Rasmussen $Y$, Smidt-JensenS, Kehlet $\mathrm{H}$, OttesenB. Fast track vaginal surgery. Acta Obstet Gynecol Scand 2002;81:138-46.

16. SjetnelS, Krogstad U, Odegard S, Engh ME. Improving quality by introducing enhanced recovery after surgery in a gynaecological department: consequences for ward nursing practice. Qual Saf Health Care 2009;18:236-40.

17. Eberhart LHJ, Koch T, Ploger B, Wagner U, Wulf H, Zwiorek $L$, et al. Enhanced recovery after major gynaecological surgery for ovarian cancer - An objective and patient-based assessment of a traditional versus a multimodal "fast track" rehabilitation programme. Anasthesiologie Und Intensiv medizin 2008;49:180-94.

18. Gerardi MA, Santillan A, Meisner B, Zahurak ML, Diaz Montes TP, Giuntoli RLII, etal. A clinical pathway for patients undergoing primary cytoreductive surgery with rectosigmoid colectomy for advanced ovarian and primary peritoneal cancers. Gynecol Oncol 2008;108:282-6.

19. Marx C, Rasmussen T, Jakobsen DH, Ottosen $C$, Lundvall $L$, Ottesen $B$, etal. The effect of accelerated rehabilitation on recovery after surgery for ovarian malignancy. Acta Obstet Gynecol Scand 2006;85:488-92.

20. Møller C, Kehlet H, Friland SG, Schouenborg LO, Lund C, Ottesen B. Fast track hysterectomy. Eur J Obstet Gynecol Reprod Biol 2001;98:18-22.

21. Egbert LD, Battit GE, Welch CE, Bartlett MK. Reduction of postoperative pain byencouragement and instruction of patients. A study of doctor-patient rapport. N Engl J Med 1964;270:82517.

22. Kiecolt-Glaser JK, PageGG, MaruchaPT, MacCallum RC, Glaser R. Psychological influences on surgical recovery. Perspectives from psychoneuroimmunology. Am Psychol 1998;53(11):1209-18.

23. Canadian Institute for Health Information. Trends in acute in patient hospitalizations and day surgery visits in Canada, 1995-1996 to 2005-2006 [database]. Ottawa(ON): Thelnstitute; 2007

24. Kalogera, E and SC Dowdy. "Enhanced Recovery Pathway in Gynecologic Surgery: Improving Outcomes Through Evidence-Based Medicine." Obstet Gynecol Clin North Am 2016;43: 551-573.

25. LiangMI,RosenMA, Rath KS, et al. Reducing re admissions after robotic surgical management of endometrial cancer: A potential for improved quality care. Gynecol Oncol.2013;131:508-511.

26. Niruthisard $S$, Werawataganon $T$, Bunburaphong $P$, Ussawanophakiat M, Wongsakornchaikul, C, Toleb, K. Improvingthe analgesic efficacy of intrathecalmorphine with parecoxib after total abdominal hysterectomy. AnesthAnalg, 2007;105:822-824.

27. Blackburn A,Stevens JD, Wheatley RG, Madej TH, Hunter D. Balanced analgesia with intravenous ketorolac and patient-controlled morphine following lower abdominal surgery. JClinAnesth 1995; 7:103-108.

28. Ong CK, Seymour RA, Lirk P, Merry AF. Combining paracetamol (acetaminophen) with nonsteroidal antiinflammatory drugs: a qualitative systematic review of analgesic efficacy for acute postoperative pain. Anesth Analg 2010; 110: 11701179.

29. Alayed N, Alghanaim N, Tan X, Tulandi T. Preemptive use of gabapentin in abdominal hysterectomy: a systematic review and meta-analysis. Obstet Gynecol, 2014;123: 1221-1229.

30. Gan TJ, Diemunsch P, Habib AS, Kovac A, Kranke P, Meyer TA, et al. Consensus guidelines for the management of postoperative nausea and vomiting. Anesth Analg. 2014;118:85-113.

31. Nelson G, Altman AD, Nick A, Meyer LA, Ramirez PT, Achtari C, Antrobus J, Huang J, Scott M, Wijk L, Acheson $\mathrm{N}$, Ljungqvist $\mathrm{O}$, DowdySC. Guidelines for pre- and intra-operative care in gynecologic/oncology surgery: Enhanced Recovery After Surgery (ERAS $\otimes$ ) Society recommendations--Part I.Gynecol Oncol. 2016;140:313-22. 


\section{Appendix 1}

\section{ERAS Protocol:}

\section{I: Preoperative diet:}

May drink clear fluids up to $2 \mathrm{~h}$ before procedure Eliminate use of mechanical bowel preparation

\section{II: Intraoperative}

\section{Analgesia before operative:}

Celecoxib $400 \mathrm{mg}$ orally once

Gabapentin 300 mg orally once

Postoperative nausea a and vomiting prophylax is Before incision (30 $\mathrm{min}$ ):

Dexamethasone $4 \mathrm{mg}$ IV once, and +/- Scopolam in epatch Before incision closure (30 $\mathrm{min}$ )

On dansetron $4 \mathrm{mg}$ IV once

\section{Fluidbalance:}

Goal: maintain intra operative euvolemia

\section{Analgesia:}

Opioids IV at discretion of anesthesiologist supplemented with ketamine, ketorolac, or both

After incision closure: injection of bupivacaine at incision site

\section{III: Postoperative}

Analgesia: Goal: no IV patient-controlled analgesia

Diet: Patient encouraged to start diet $4 \mathrm{~h}$ after procedure

Activities: Evening of surgery: out of bed greater than $2 \mathrm{~h}$, including one or more walks and sitting in chair Patient up in chair for all meals

Fluid balance: Operating room fluids discontinued on arrival to floor 